Technische

Universität

Berlin

\author{
Moritz Sieber, Christian Oliver Paschereit, Kilian Oberleithner
}

\title{
Advanced identification of coherent structures in swirl-stabilized combustors
}

Open Access via institutional repository of Technische Universität Berlin

\section{Document type}

Journal article | Accepted version

(i. e. final author-created version that incorporates referee comments and is the version accepted for publication; also known as: Author's Accepted Manuscript (AAM), Final Draft, Postprint)

This version is available at

https://doi.org/10.14279/depositonce-12717

Citation details

Sieber, M., Oliver Paschereit, C., Oberleithner, K. (2016). Advanced Identification of Coherent Structures in Swirl-Stabilized Combustors. In Journal of Engineering for Gas Turbines and Power (Vol. 139, Issue 2). ASME International. https://doi.org/10.1115/1.4034261.

Terms of use

This work is protected by copyright and/or related rights. You are free to use this work in any way permitted by the copyright and related rights legislation that applies to your usage. For other uses, you must obtain permission from the rights-holder(s). 


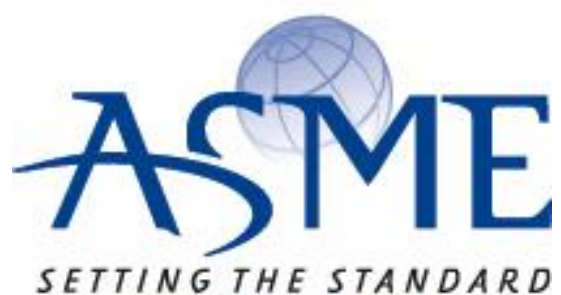

SETTING THE STANDARD

\section{American Society of Mechanical Engineers}

ASME Accepted Manuscript Repository

Institutional Repository Cover Sheet

ASME Paper Title: Advanced Identification of Coherent Structures in Swirl-Stabilized Combustors

Authors: $\quad$ Moritz Sieber, Christian Oliver Paschereit, Kilian Oberleithner

ASME Journal Title: Journal of Engineering for Gas Turbines and Power

Volume/Issue

139(2): 021503 (8 pages) Date of Publication (VOR* Online) September 13, 2016

ASME Digital Collection URL: https://asmedigitalcollection.asme.org/gasturbinespower/

article-abstract/139/2/021503/374397/

DOI: $\quad$ https://doi.org/10.1115/1.4034261

*VOR (version of record) 


\title{
ADVANCED IDENTIFICATION OF COHERENT STRUCTURES IN SWIRL-STABILIZED COMBUSTORS
}

\author{
Moritz Sieber; Christian Oliver Paschereit and Kilian Oberleithner \\ Chair of Fluid Dynamics \\ - Hermann-Föttinger-Institut - \\ Technische Universität Berlin \\ Müller-Breslau-Str. 8, 10623 Berlin, Germany
}

\begin{abstract}
We present an application of a newly introduced method to analyze the time-resolved experimental data from the flow field of a swirl-stabilized combustor. This method is based on classic proper orthogonal decomposition (POD) extended by a temporal constraint. The filter operation embedded in this method allows for continuous fading from the classic POD to the Fourier mode decomposition. This new method - called spectral proper orthogonal decomposition (SPOD) - allows for a clearer separation of the dominant mechanisms due to a clean spectral separation of phenomena. In this paper, the fundamentals of SPOD are shortly introduced. The actual focus is put on the application to a combustor flow. We analyze high-speed PIV measurements from flow fields in a combustor at different operation conditions. In these measurements, we consider externally actuated, as well as natural dynamics and reveal how the natural and actuated modes interact with each other. As shown in the paper, SPOD provides detailed insight into coherent structures in swirl flames. Two distinct PVC structures are found that are very differently affected by acoustic actuation. The coherent structures are related to heat release fluctuations, which are derived from simultaneously acquired $\mathrm{OH}^{*}$ chemiluminescence measurements. Besides the actuated modes, a low frequency mode was found that significantly contribute to the global heat release fluctuations.
\end{abstract}

\footnotetext{
*Address all correspondence to this author: moritz.sieber@tu-berlin.de
}

\author{
NOMENCLATURE \\ a Temporal SPOD mode coefficients \\ $c$ Correlation coefficient \\ $g \quad$ SPOD filter coefficients \\ I $\mathrm{OH}^{*}$ chemiluminescence intensity \\ $\lambda$ SPOD mode energy \\ $M \quad$ Number of spatial points \\ $N$ Number of snapshots \\ $N_{f} \quad$ Size of the SPOD filter \\ $\Omega \quad$ Spatial SPOD mode (chemiluminescence) \\ $\Phi \quad$ Spatial SPOD mode (velocity) \\ $q$ Global heat release \\ R Correlation matrix \\ S Filtered correlation matrix \\ St Strouhal number \\ $t$ Time \\ $\mathbf{v}$ Cartesian velocity vector $[u, v, w]^{T}$ \\ $\mathbf{x}$ Cartesian coordinate vector $[x, y, z]^{T}$
}

\section{INTRODUCTION}

It's getting increasingly harder to identify the relevant information from the vast amount of data that is produced from recent measurement systems or computational fluid dynamics. Beside the mean quantities, the available time-resolved data put the focus towards dynamic phenomena in the flows. The dynamics of interest are usually related to large-scale periodic structures, also called coherent structures [1]. These are of major importance in 
gas turbine combustors, since they interact with the flame and modulate the heat release of the combustor $[2,3]$.

In data of combustor flows and turbulent flows in general, the identification of coherent structures is aggravated by the occurrence of multiple different structure. Several dynamic structures may occur simultaneously, interact with each other and dis/appear intermittently. In order to extract these structures from the data, modal decompositions are commonly used. There are two different approaches that can be employed to build such a modal basis for flow data. One of them is the POD [1], which provides a basis of modes that is optimal in terms of energy representation. The other approach includes the dynamic mode decomposition (DMD) [4,5] or classical Fourier mode decomposition, where each mode is assigned to a single frequency. Both approaches are problematic when highly turbulent flows are investigated. The POD puts a strict focus on the maximization of the represented energy of a mode without any specification on the dynamic of these modes. This may result in the merging of multiple (e.g. less energetic) dynamical structures in a single POD mode. Moreover, the POD modes strongly depend on the snapshot ensemble and may differ between subsequent measurements, which hinder the comparison of decompositions from different measurements. The restriction to constant frequencies of single modes (DMD, Fourier) can eliminate this ambiguity, but at the cost of a much larger set of modes. Even slight frequency variations of a single flow structure are reflected by several modes at neighboring frequencies. In consequence, the modes represent less energy, resulting in lower signal to noise ratios of each single mode, which produces much noisier spatial modes.

An escape from this dilemma is provided by the spectral proper orthogonal decomposition (SPOD) [6], which unifies both approaches and allows for a continuous transition from the POD to the Fourier mode decomposition. Employing a soft spectral constraint, the SPOD permits frequency variations of single modes, while it still separates modes according to their spectral contents. The SPOD acts somewhat like a band-pass filter to the POD modes, but the bands are selected intrinsically by the flow dynamics.

In this paper, the SPOD is applied to Particle Image Velocimetry (PIV) data of a swirl-stabilized combustor. Natural flow dynamics as well as flows actuated at a constant frequency and several amplitudes are considered. Furthermore, a strategy is presented, which allows the inclusion of data that are measured simultaneously with the velocity. Here, data from $\mathrm{OH}^{*}$ chemiluminescence is used to demonstrate the procedure. The combined analysis of coherent velocity fluctuations and heat release rate fluctuations reveal the connection between the flame describing function and coherent structures.

This paper starts with the explanation of the experimental setup, followed by a description of the applied mathematical methods and the results obtained from the application of

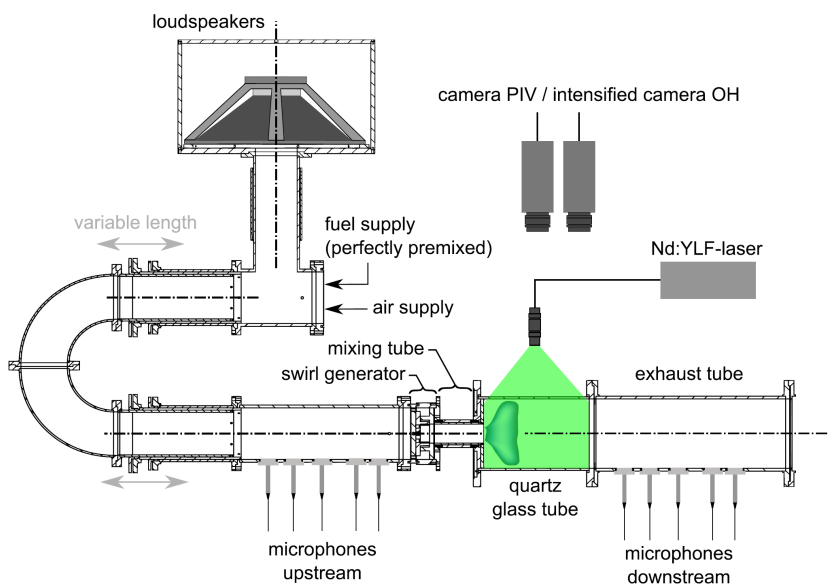

FIGURE 1. Sketch of the atmospheric combustion test-rig and measurement instrumentation

these methods. The final conclusions highlight the main findings gained from the application of SPOD to this highly turbulent reacting flow.

\section{EXPERIMENTAL SETUP Experimental Facility}

The experimental data presented in this paper are obtained from an atmospheric combustion test-rig. A sketch of this setup together with the experimental apparatus is presented in fig. 1. A swirl-stabilized combustor is investigated at perfectly premixed conditions. Swirl is generated by injecting fluid tangentially into a mixing tube that terminates in the combustion chamber. For the considered experiments, the supplied air mass-flow is $100 \mathrm{~kg} / \mathrm{h}$, which results in a Reynolds number of around $\mathrm{Re}=60$ 000. The supplied air is not preheated. The combustor is operated with methane (natural gas) at an equivalence ratio of 0.7 .

The test-rig allows for the actuation of streamwise acoustic waves using loudspeakers mounted upstream of the burner. In the present investigation, a forcing with constant frequency at different amplitudes is presented. The different amplitudes correspond to a modulation of the total flow by 10,40 and $70 \%$ of the mean flow at the inlet $\left(\left|u^{\prime}\right| / \bar{u}\right)$. The actuation frequency is $268 \mathrm{~Hz}$, which corresponds approximately to the maximum flame response at this operation conditions, according to measurements with the multi-microphone method [7].

\section{Data Acquisition}

Inside the combustion chamber, the velocities and $\mathrm{OH}^{*}$ chemiluminescence were recorded simultaneously with two synchronized high-speed cameras. Optical access to the combustion 
chamber was enabled through a $200 \mathrm{~mm}$ diameter quartz glass pipe confining the flame.

Flow measurements were conducted by means of PIV in the meridional section as indicated in the schematic. The PIV system consists of a Photron Fastcam SA 1.1 high-speed camera (1Mpixel at $2.7 \mathrm{kHz}$ double frame) and a Quantronix Darwin Duo laser $(30 \mathrm{~mJ}$ at $1 \mathrm{kHz})$. For each configuration, a set of 2000 images was recorded at a rate of $2000 \mathrm{~Hz}$. The PIV data were processed with PIVview (PIVTEC GmbH) using standard digital PIV processing [8] enhanced by iterative multigrid interrogation [9] with image deformation [10].

The chemiluminescence of the $\mathrm{OH}^{*}$ radicals was simultaneously recorded with a second Photron Fastcam SA 1.1. This was equipped with an image intensifier and an optical filter that restricts the observed wavelengths to $295-340 \mathrm{~nm}$.

\section{DATA ANALYSIS METHODOLOGY \\ Spectral Proper Orthogonal Decomposition}

The recently introduced SPOD is used to analyze the velocity and identify the coherent structures that occur in the considered flow. A detailed derivation and discussion of the SPOD can be found in [6]. Here, only the practical implementation is described. The SPOD is an offspring of the classical POD. Holmes et al. [1] provide full details on POD and recent publications from our group discuss the method in the context of swirling jets $[11,12]$. The application and computation of SPOD is generally comparable to classical POD, although there are some modifications in order to control the spectral properties of the decomposition.

The starting point of the SPOD is a decomposition of the velocity vector $\mathbf{v}$ into a mean $\overline{\mathbf{v}}$ and a fluctuating part $\mathbf{v}^{\prime}$ that is represented as a series, reading

$$
\mathbf{v}(\mathbf{x}, t)=\overline{\mathbf{v}}(\mathbf{x})+\sum_{i=1}^{N} a_{i}(t) \Phi_{i}(\mathbf{x})
$$

where $\mathbf{x}$ denotes the coordinate vector and $t$ the time. The decomposition consists of $a_{i}$ temporal coefficients and $\Phi_{i}$ spatial modes.

A set of $M$ spatial points recorded simultaneously over $N$ time steps is considered. To calculate the SPOD, the correlation matrix of this data set is needed, which is calculated between individual snapshots (temporal correlation). This is calculated from an appropriate inner product $\langle$,$\rangle , defined as$

$$
\langle\mathbf{u}(\mathbf{x}), \mathbf{v}(\mathbf{x})\rangle=\int_{V} \mathbf{u}(\mathbf{x}) \mathbf{v}^{T}(\mathbf{x}) \mathrm{d} V
$$

where $V$ specifies the spatial region or volume over which the correlation is integrated. The elements of the correlation matrix
$\mathbf{R}$ are given by

$$
R_{i, j}=\frac{1}{N}\left\langle\mathbf{v}^{\prime}\left(\mathbf{x}, t_{i}\right), \mathbf{v}^{\prime}\left(\mathbf{x}, t_{j}\right)\right\rangle
$$

Matrix $\mathbf{R}$ is of size $N \times N$.

In contrast to the classical POD algorithm, a filter is inserted at this point, which modifies the correlation matrix $\mathbf{R}$. To augment the diagonal similarity of $\mathbf{R}$, a simple low-pass filter is applied along the diagonals. This results in a filtered correlation matrix $\mathbf{S}$, with the elements given as

$$
S_{i, j}=\sum_{k=-N_{f}}^{N_{f}} g_{k} R_{i+k, j+k} .
$$

The filter above is just a symmetric finite impulse response filter with a filter coefficients vector $\mathbf{g}$ of length $2 N_{f}+1$. The most simple approach would be a box filter, where all coefficients have the same value $g_{k}=\frac{1}{2 N_{f}+1}$. In this article, we use a Gaussian filter, which features a smooth response in time and frequency domain. Moreover, we choose a standard deviation as such that the filter gives the same cut-off frequency as a box filter with half the length.

The temporal coefficients $\mathbf{a}_{i}=\left[a_{i}\left(t_{1}\right), \ldots, a_{i}\left(t_{N}\right)\right]^{T}$ and mode energies $\lambda_{i}$ are obtained from the eigenvectors and eigenvalues of the filtered correlation matrix.

$$
\mathbf{S a}_{i}=\lambda_{i} \mathbf{a}_{i} ; \quad \lambda_{1} \geq \lambda_{2} \geq \cdots \geq \lambda_{N} \geq 0
$$

The subscript $i$ refers to single eigenvalues, which are sorted in descending order. The eigenvectors $\mathbf{a}_{i}$ of $\mathbf{S}$ are orthogonal and they are scaled with the energy of the single modes such that

$$
\frac{1}{N}\left(\mathbf{a}_{i}, \mathbf{a}_{j}\right)=\lambda_{i} \delta_{i j}
$$

where $($,$) denotes the scalar product and \delta_{i j}$ the Kronecker delta. The spatial modes are obtained from the projection of the snapshots onto the temporal coefficients

$$
\Phi_{i}(\mathbf{x})=\frac{1}{N \lambda_{i}} \sum_{j=1}^{N} a_{i}\left(t_{j}\right) \mathbf{v}^{\prime}\left(\mathbf{x}, t_{j}\right)
$$

This spatial mode base is orthogonal if $N_{f}=0$, which resembles the classic POD. The filter size $N_{f}$ controls the spectral bandwidth of the individual modes, which allows a continuous transition from the classical POD to the Fourier decomposition. Thus, 
the lower $\left(N_{f}=0\right)$ and upper $\left(N_{f}=N\right)$ bound of the filter width correspond to the POD and Fourier decomposition, respectively. The best results are obtained when the filter width matches a characteristic time-scale of the flow. This can either be the period of a dominant oscillation or the characteristic length-scale divided by the characteristic velocity-scale.

The SPOD provides natural sorting of the spatial modes in terms of their turbulent kinetic energy $\lambda_{i}$. Another ranking is introduced by Sieber et al. [6] that is based on the cross spectra of mode pairs. This approach is based on the fact that a periodic coherent structure appears as a pair of modes in the decomposition. These pairs are identified by their level spectral coherence, which also delivers a ranking of periodic coherent structures.

\section{Extended SPOD of $\mathrm{OH}^{\star}$ Chemiluminescence Data}

Additional quantities that are recorded simultaneously with the velocities can also be represented in the modal basis of the SPOD. A simple way to accomplish this is the consideration of these quantities together with the velocity in an extended state vector of the flow. However, this approach requires a consistent implementation of these quantities into the inner product [13]. For the present case, a common measure for velocities measured within a planar field and line-integrated $\mathrm{OH}^{*}$ chemiluminescence must be found. Since this would only allow for a flawed measure, we pursue another approach.

The extended POD introduced by Boree [14] enables to represent any kind of simultaneous measurement in the same basis as the POD. This approach can be similarly applied to the SPOD as will be shown here. The intensity of the $\mathrm{OH}^{*}$ chemiluminescence $I$ is decomposed into a mean part $\bar{I}$ and a fluctuating part $I^{\prime}$ that is represented as a series, reading

$$
I(\mathbf{x}, t)=\bar{I}(\mathbf{x})+I^{\prime}(\mathbf{x}, t)=\bar{I}(\mathbf{x})+\sum_{i=1}^{N} a_{i}(t) \Omega_{i}(\mathbf{x}) .
$$

Here, the same expansion coefficients $a_{i}$ are used that are derived from the decomposition of the velocity field. Consequently, the $\mathrm{OH}^{*}$ modes $\Omega_{i}$ are also obtained by the projection of the $\mathrm{OH}^{*}$ snapshots onto the coefficients, with

$$
\Omega_{i}(\mathbf{x})=\frac{1}{N \lambda_{i}} \sum_{j=1}^{N} a_{i}\left(t_{j}\right) I^{\prime}\left(\mathbf{x}, t_{j}\right) .
$$

The spatial points $\mathbf{x}$ do not require to be the same as those for which the velocity was measured. Moreover, the extended SPOD can also be applied to any other kind of simultaneous measurement.

\section{Correlation of Global Heat Release and Coherent Structures}

The relation between the heat release and the coherent structures is quantified by a correlation measure. The global heat release $q$ is obtained from the spatially integrated $\mathrm{OH}^{*}$ chemiluminescence, with

$$
q(t)=\int_{A} I(\mathbf{x}, t) \mathrm{d} A
$$

where $A$ specifies the spatial integration area, which is taken to be the entire measured domain. The heat release is also represented by a mean and fluctuation part

$$
q(t)=\bar{q}+q^{\prime}(t)
$$

The fluctuating part is correlated with the SPOD expansion coefficients to measure the contribution of the individual modes to the heat release fluctuations. It is given by

$$
c_{i}=\frac{1}{N \sqrt{\lambda_{i}}} \sum_{j=1}^{N} a_{i}\left(t_{j}\right) q^{\prime}\left(t_{j}\right) .
$$

In fact, this is just the extended SPOD of a single point signal.

\section{RESULTS \\ The Mean Flow}

The time-averaged flow is considered for the natural case without any actuation. The velocity field is depicted in fig. 2 . It shows a typical conical vortex breakdown shape that corresponds to a V-flame $[15,16]$. The crosswise extend of the measured domain reaches almost up to the combustor side walls, and the attachment (and deflection) of the jet at the wall is visible. The velocity field shows a large recirculation zone inside the conical jet. At the upstream end, this is delimited by a stagnation point on the jet axis. Two additional recirculation zones develop between the jet and the outer confinement.

In fig. 3, the mean $\mathrm{OH}^{*}$ intensity is depicted. An inverse Abel transformation is used to deduce the intensity in a plane from the line integrated intensities. Therefore, a rotational symmetry of the field is assumed. The $\mathrm{OH}^{*}$ distribution shows that this configuration features a V-shaped flame, which is anchored in the inner shear-layer shortly downstream of the combustor inlet.

\section{The Coherent Structures of the Natural Flow}

The classical POD and the SPOD of the natural flow are contrasted in this section. This demonstrates best the increased performance of the SPOD. Both decompositions are calculated using the algorithm explained above, but with different filter widths 


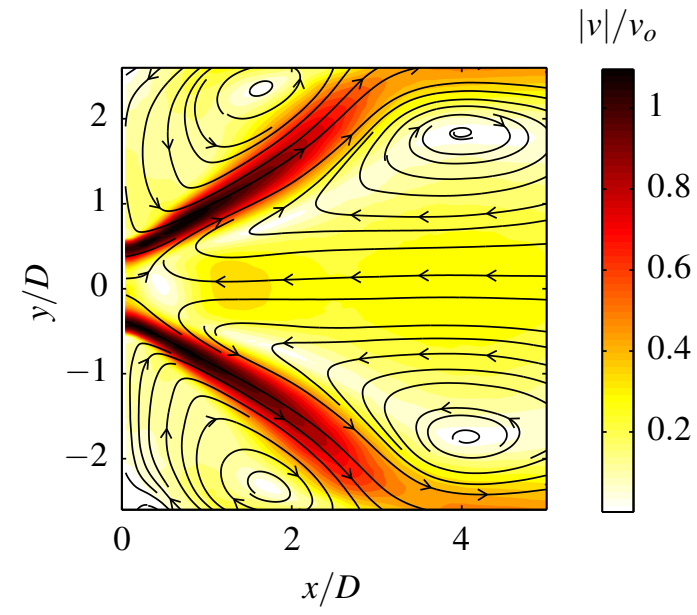

FIGURE 2. Natural mean velocity field (magnitude and streamlines)

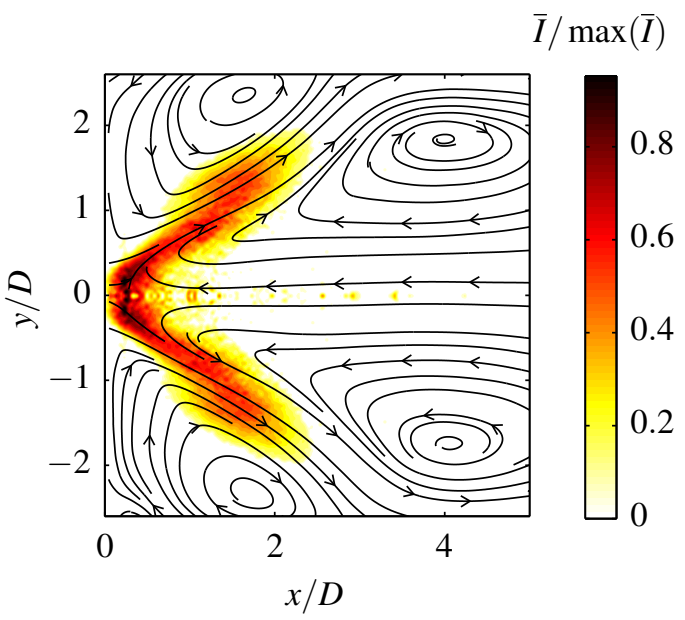

FIGURE 3. Natural mean $\mathrm{OH}^{*}$-chemiluminescence field (de-Abeled) with superimposed streamlines

$N_{f}$. In the SPOD scheme, the classic POD refers to a vanishing filter $N_{f}=0$. For the SPOD shown here we choose a filter width of $N_{f}=24$, which is equivalent to the period of the dominant oscillation with the lowest frequency $(\mathrm{St}=0.1)$. It is calculated from the ratio of acquisition frequency $f_{a}$ to mode frequency $f_{m}$ as $N_{f}=f_{a} / f_{m}$. The decomposition is calculated only from the crosswise velocity (y-direction) since this has proven to give the best indication of coherent structures in this flow. The addition of the streamwise velocity puts the focus more towards largescale variations of the base flow, which will be misleading in this context.

In conjunction with the crosswise velocity, the shape of symmetric and anti-symmetric structures in the rotational symmetric flow shall be clarified. The structures that are discussed in this article are the Precessing Vortex Core (PVC) [15] and the ac-
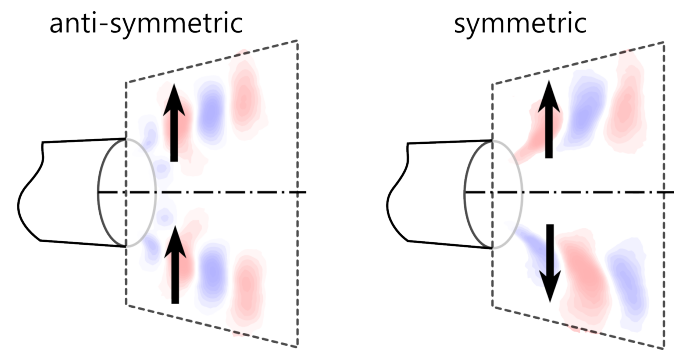

FIGURE 4. Relation between the sign of the crosswise velocities and the mode symmetries

tuated mode. The PVC is a helical mode, which appears as an anti-symmetric structure in the measured section. The actuated mode is rotationally symmetric and therefore also symmetric in the measured plane. However, the sign of the crosswise velocity shows an opposed symmetry as depicted in fig. 4.

The POD and SPOD of the natural flow are given in fig. 5 and fig. 6 respectively. For both decompositions, the first seven most energetic modes are drawn in descending order. They are represented by the power spectral density (PSD) of the mode coefficient $a_{i}$ (left) and the contour plot of the spatial mode $\Phi_{i}$ (right). The spatial modes are displayed together with the streamlines of the mean flow to allow for a better orientation.

The first pair of POD modes (fig. 5) show a similar structure that resembles the type I PVC identified in ref. [15] or the global mode reported in ref. [11]. The wavelength of this structure and the corresponding Strouhal number are very similar to those observed in other swirling flows. However, the mode coefficients spectrum shown here is considerably broader indicating low oscillation amplitudes, which is due to the specific design of this swirler [16]. The mode is located close to the combustor inlet and describes a periodic displacement of the conical jet, which is related to the precessing motion of the vortex core (PVC). Considering the remaining modes, we see what we call a "mode clutter". The spatial modes reveal multiple wavelengths with no clear symmetries and the spectra reveal no distinct peaks. In the spectra of the modes 5 to 7 , there is a peak at $S t \approx 0.1$, which might indicate another dominant structure. However, it is not properly assigned to a single mode pair.

The SPOD decomposition gives a more ordered and clearer set of modes (fig. 6). Obviously, the applied filter restricts the spectral bandwidth of the individual modes, which results in well-defined spectral peaks of the mode coefficients. The PVC-I mode already found in the POD, is represented by mode 1 and 2 , with a similar shape as for the POD, but with a well-defined peak in the spectrum. The 3rd and 4th SPOD modes also describe a single structure, which resembles the type II PCV identified in ref. [15]. The spectral peak at $\mathrm{St}=0.1$ as well as its spatial location inside the recirculation zone matches with the previous observations. However, the spatial structure of the PVC-II does 

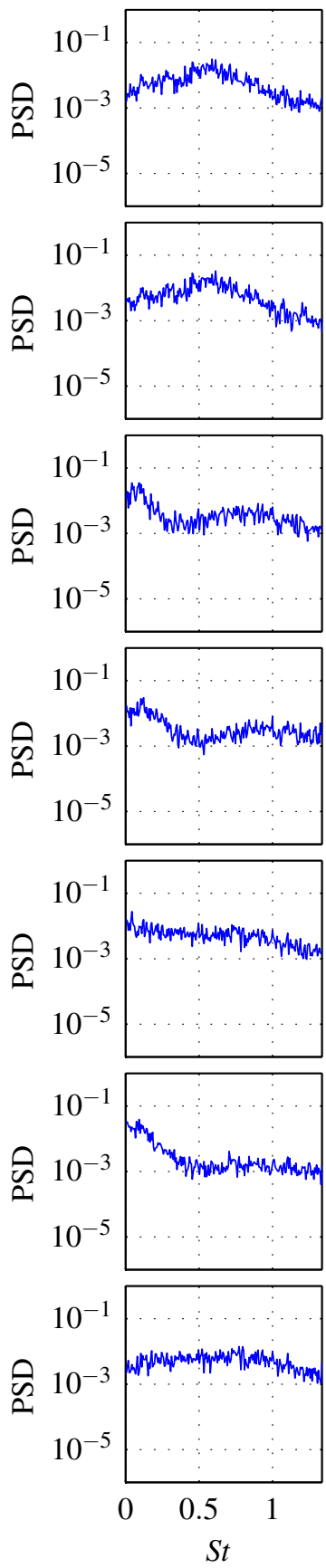

FIGURE 5. POD $\left(N_{f}=0\right)$ modes of the natural flow.

not show such a proper anti-symmetry as the PVC-I. This is attributed to the very low frequencies, and hence, insufficient statistical convergence. In this context, it is also visible that the spatial development of this mode is limited by the confinement of the combustion chamber.

The SPOD modes 6 and 7 are also coupled, but their spec-
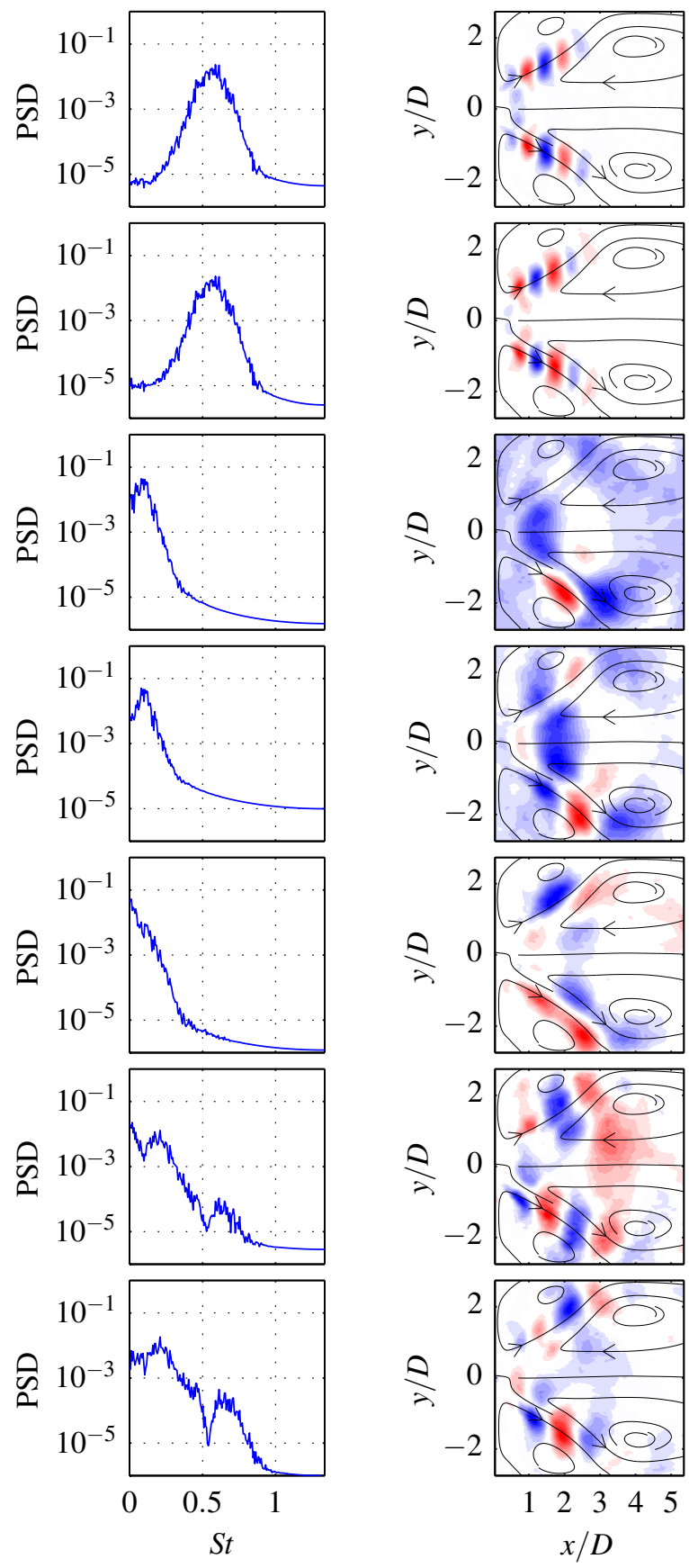

FIGURE 6. $\operatorname{SPOD}\left(N_{f}=24\right)$ modes of the natural flow.

tra have two peaks close to the two other modes and their spatial wavelength lies in between the other two PVC modes. The 5th mode, however, has no counterpart. It shows low frequency temporal dynamics and the spatial mode tends towards a symmetric structure. It might be a so-called shift mode $[12,17]$ that describes slow changes of the mean velocity field. 
This comparison of POD and SPOD shows that the additional spectral constraint of SPOD creates an interpretable set of modes, where individual phenomena are clearly assigned to single modes or mode pairs.

\section{Impact of Actuation on the Coherent Structures}

The coherent structures are now identified from the measurements at increasing forcing amplitudes. The SPOD of the individual measurements are presented in fig. 7 using a compact presentation-scheme introduced in ref. [6]. A single configuration is represented by the so-called SPOD spectrum (see the scatter plot on the left), where each dot represents a pair of modes that is linked to the spectral coherence of each mode. The level of spectral coherence is indicated by the diameter and color of the dots. On the right-hand side, next to the SPOD spectrum, the spatial and temporal properties of selected modes are plotted. The selection is based on the SPOD spectrum and the modes are labeled by numbers (\#1-4) in the SPOD spectrum as well as between the mode plots. The upper row displays the spatial structure of the modes $\left(\Phi_{i}\right)$ and the lower row the frequency spectrum (PSD) of the corresponding mode coefficients $\left(a_{i}\right)$.

The uppermost decomposition is the same as the one presented in the previous section (fig. 6). The SPOD spectrum clearly highlights the PVC-I (\#1) and -II (\#2) identified above. Moreover, mode \#4 was also identified before and the additional mode \#3 lies close to PVC-I. It shows two spectral peaks right above and below the Strouhal number of PVC-I and its spatial shape partially resembles the one of PVC-I.

The second configuration presented in the second row of fig. 7 corresponds to "weak" actuation, with a forcing amplitude of $10 \%$ of the mean flow. The two PVC modes (\#1 and \#2) stay nearly unaltered, but the SPOD spectrum indicates another mode that appears right at the forcing frequency of $S t=0.35$ (\#3). This mode exhibits a constant frequency and a symmetric spatial structure, corresponding to the symmetric forcing. The additional mode \#4 may be related to mode \#3 and \#4 of the natural case. This mode is less energetic and shows weaker spectral coherence as can be seen from the SPOD spectrum.

In the configuration considered next, the forcing amplitude is increased considerably to $40 \%$ of the mean flow (see third row of fig. 7). This strongly alters the energy distribution among the observed modes. The forcing mode (\#1) now clearly contributes most to the fluctuations in the flow, whereas the energy of the PVC-I mode (\#4) is significantly reduced. The PVC-II mode (\#2) stays at the same energy, but its spatial structure is altered. At the first harmonic of the forcing frequency, another mode appears (\#3), with half the spatial wavelength and the same symmetry as the forcing mode.

For the last considered case, the forcing is increased to $70 \%$ of the mean flow (bottom row of fig. 7). The forcing mode (\#1) together with its first (\#4) and second harmonic broadly dominate the SPOD spectrum. The only other modes the are distinct in the SPOD spectrum is the low frequency PVC-II and another mode (\#3) that exhibits a somewhat higher frequency and spatial wavelengths than the PVC-II.

The SPOD allows for identifying the same coherent structures for every actuation case, and reveals the impact of the actuation on the entire mode spectrum. The three outstanding structures observed for the different measurements are the PVC-I, -II and the actuation mode. As expected, the energy of the actuation mode raises with increasing actuation amplitude. Moreover, the low frequency PVC-II mode seems little affected by the growing actuation. The energy only slightly reduces and the spatial structure changes a little. The PVC-I mode, however, does not take the actuation undisturbed. It is strongly reduced in energy until it entirely vanishes for the maximum actuation level. If the evolution of the entire mode spectrum is considered, it is visible that the actuation modes (fundamental together with the higher harmonics) dominate the high frequency part of the spectrum. All the modes with weaker energy are attenuated in the spectrum. Hence, the fluctuation energy in the flow is redistributed from the broad turbulent structures to the actuated structure and the higher harmonics. The modes with frequencies below the actuation are only weakly influenced by the actuation.

\section{Spatial $\mathrm{OH}^{\star}$ Chemiluminescence Structures}

The configuration with $10 \%$ actuation amplitude is selected for further investigations with respect to the $\mathrm{OH}^{*}$ chemiluminescence. This case is considered, because it features all three major structures identified so far, namely the PVC-I, -II and the actuated mode. Fig. 8 shows the corresponding $\mathrm{OH}^{*}$ modes as computed from an extended SPOD.

It is worth noting that for all three modes, the wavelengths of the extended SPOD $\mathrm{OH}^{*}$ modes do not match with those of the velocity modes. The $\mathrm{OH}^{*}$ modes show line integrated information that cannot directly be compared to the planar velocity data. A tomographic reconstruction of the $\mathrm{OH}^{*}$ mode filed would be necessary to allow a direct comparison [3]. However, all the $\mathrm{OH}^{*}$ modes show clear structures that feature the same spatial symmetries as the velocity modes (recall fig. 4). Moreover, the velocity and $\mathrm{OH}^{*}$ modes both cover the same spatial areas. This shows that the extended SPOD is well suited to reveal the spatial heat release rate distribution corresponding to the coherent vortex structures in the flow field.

\section{Relation of Global Heat Release and Flow Structures}

In order to quantify, which coherent structure contributes to the global heat release rate fluctuations in the combustor, the integrated chemiluminescence $q^{\prime}$ is correlated with the mode coefficients $a_{i}$, as expressed in eq. (12). For this purpose, the SPOD is computed from the symmetric part of the velocity field as described in [12]. The symmetric part is calculated from the sum 

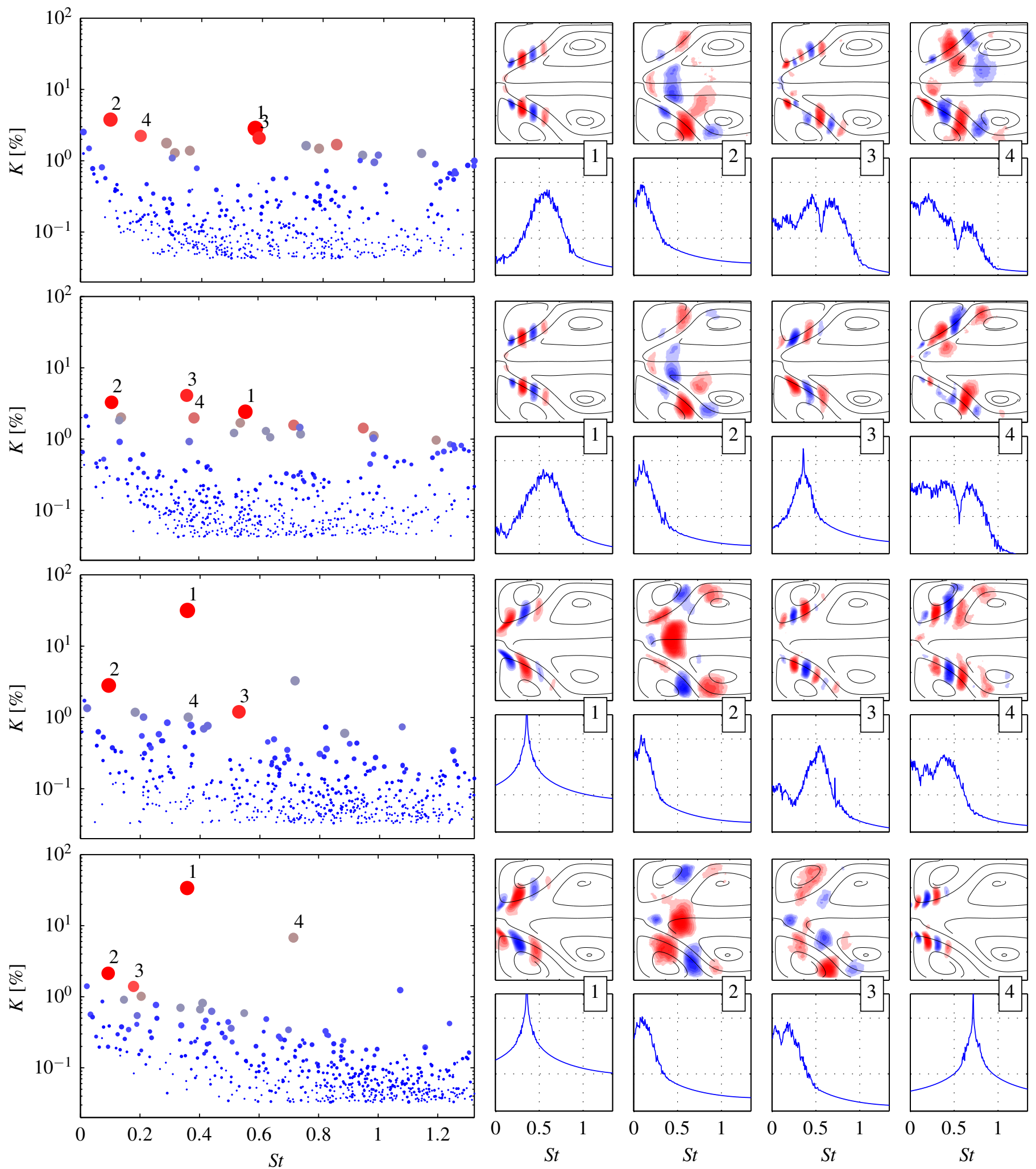

FIGURE 7. SPOD spectrum (left) and spatial modes with mode coefficient spectrum (right) for increasing forcing amplitudes (from top to bottom the forcing amplitudes are $0,10,40$ and $70 \%$ of the bulk velocity) 

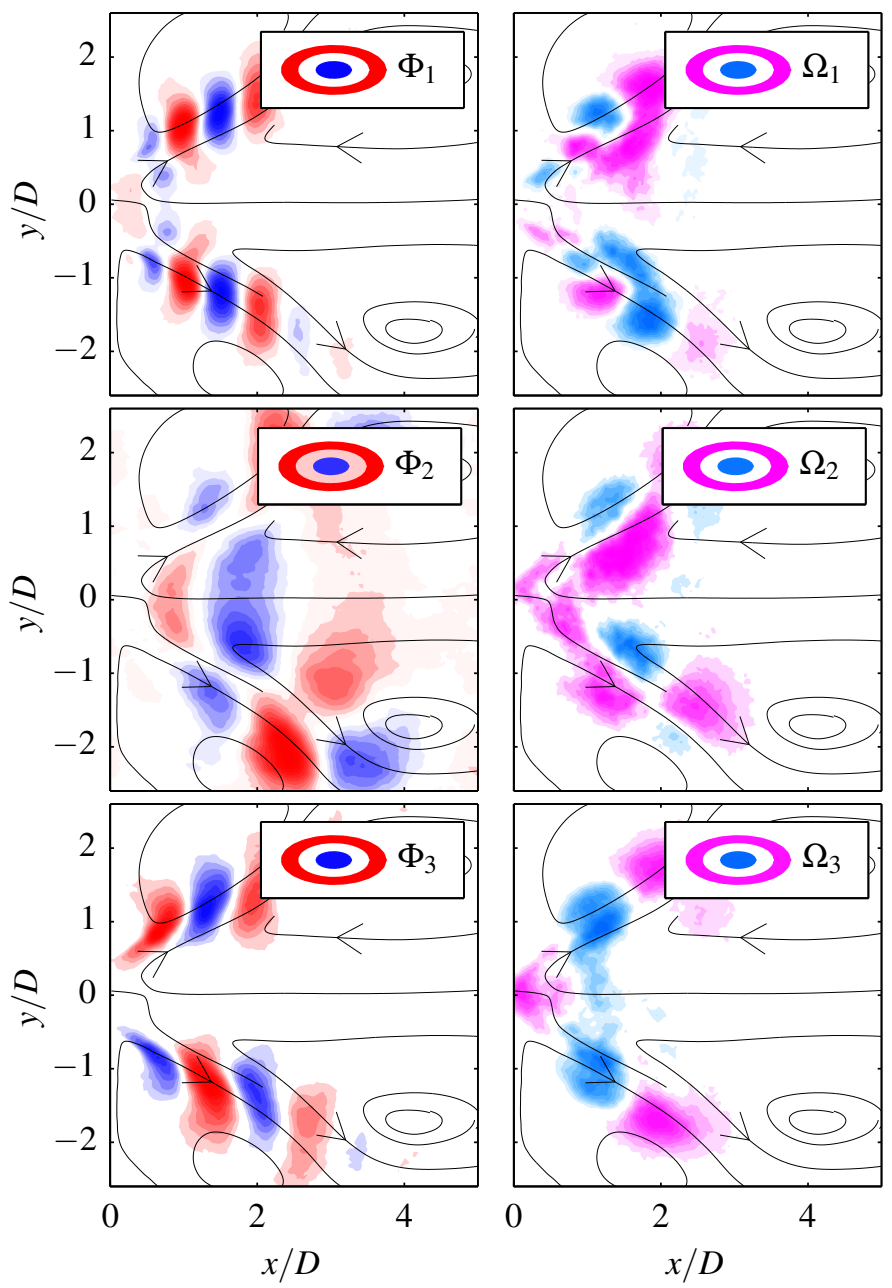

FIGURE 8. SPOD velocity modes $\Phi_{i}$ and the corresponding SPOD $\mathrm{OH}^{*}$ modes $\Omega_{i}$ for the dominant coherent structures

of the original field and the field mirrored at the x-axis. The reasoning behind this operation is that the anti-symmetric velocity fluctuations may only cause anti-symmetric heat release rate fluctuations that do not contribute to the integral heat release. Therefore, they are excluded from the analysis right from the beginning on.

Not surprisingly, the correlation reveals that the forced axissymmetric mode contributes most to the global heat release rate fluctuations (not shown). The periodic vortex shedding deforms the flame and modulates the heat release rate. This interaction is also evident from fig. 8. Regardless of the actuation amplitude, the actuated mode is the only mode that shows significant correlation with the heat release rate.

The consideration of the natural case delivers more interesting results. We find one low frequency mode that shows significant correlation with $q^{\prime}$. The coefficient of this mode together with the global heat release rate fluctuations is given in fig. 9. It becomes visible that the low frequency variations of $q^{\prime}$ follows the same trend as the mode coefficient $a_{i}$. It is also evident that only a part of the global heat release rate fluctuations can be explained by this mode.

The spatial structure of this correlated mode together with the corresponding extended SPOD OH* mode is given in fig. 10 . The velocity mode is comparable to a mode found in the investigation of a generic unconfined swirling jet [12]. There, the mode corresponds to an axial shift of the recirculation region (upstream stagnation point), which is related to fluctuations of the swirl intensity. Here, this shift mode also describes very low frequency mean flow unsteadiness $(0<S t<0.01)$ and shows a similar spatial structure. The corresponding $\mathrm{OH}^{*}$ mode shows an axis-symmetric shape and high amplitudes at the upstream end of the recirculation zone, which indicates axial fluctuations of the flame root.

Combining these findings, the analysis shows that the low frequency variations of the velocity field affect the flame root and thereby cause variations of the global heat release. This result indicates that the low frequency part of the flame describing function is governed by changes of the mean velocity field and not by hydrodynamic instabilities. In contrast, the actuation causes periodic vortex shedding, which strongly depends on the hydrodynamic stability of the base flow, as detailed by Oberleithner et al. [18].

\section{CONCLUSIONS}

The newly introduced SPOD is demonstrated for measurement data of a swirl-stabilized combustor, where it precisely identifies all the dominant coherent structures in the flow. Two different PVC structures are identified in the same flow configuration, which were previously only separately observed in different configurations.

With the help of the SPOD, the coherent structures of four different sets could be compared in a consistent manner. The robust identification of the dominant coherent structures allows for tracking the evolution of these structures across the measurements. This allows for quantifying the effect of the actuation on all the coherent structures that are present in the flow and not only on the actuated structure alone. The analysis shows that the actuated mode suppresses the PVC-I mode, whereas the PVC-II mode stays remarkably unaffected.

Employing the extended SPOD, the $\mathrm{OH}^{*}$ chemiluminescence measurements are related to the identified coherent structures. It appears that all the identified structures are relevant for spatial fluctuations of the heat release rate. A separate consideration of the integral $\mathrm{OH}^{*}$ data together with symmetric velocity fluctuations shows that low frequency variations of the recirculation region transfers to fluctuations of the global heat release. The results indicate that the pathway from velocity fluctuations to heat release fluctuations, which 


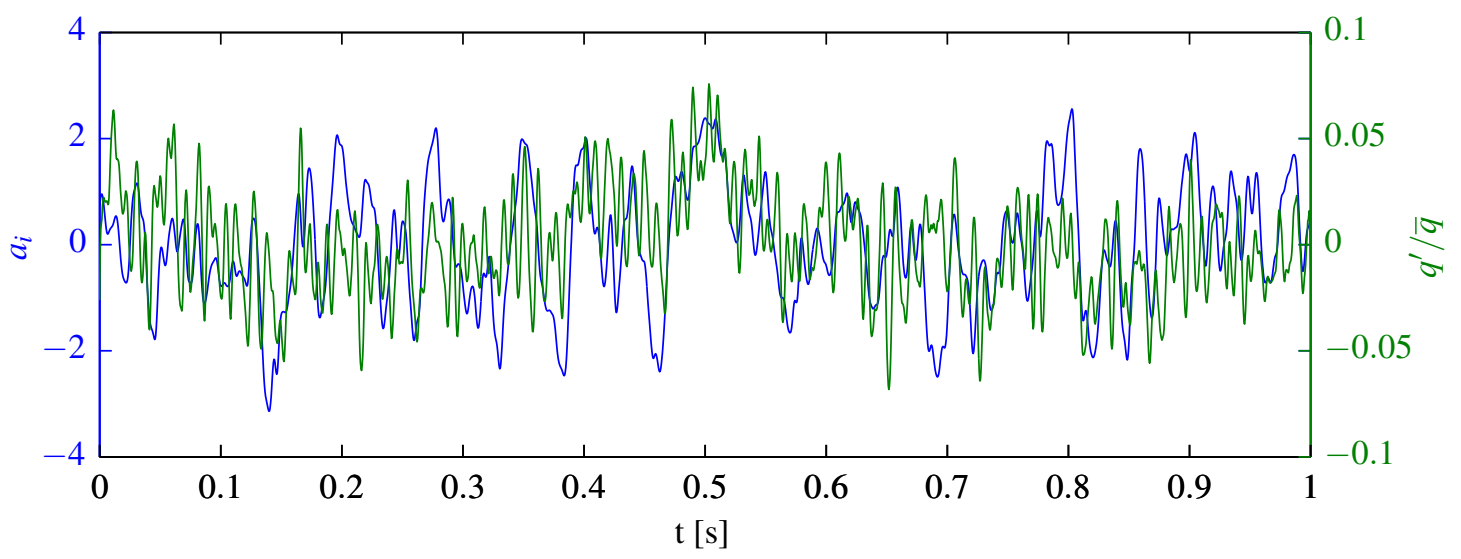

FIGURE 9. Time curve of the SPOD coefficient $\left(a_{i}\right)$ and the global heat release fluctuations $\left(q^{\prime} / \bar{q}\right)$

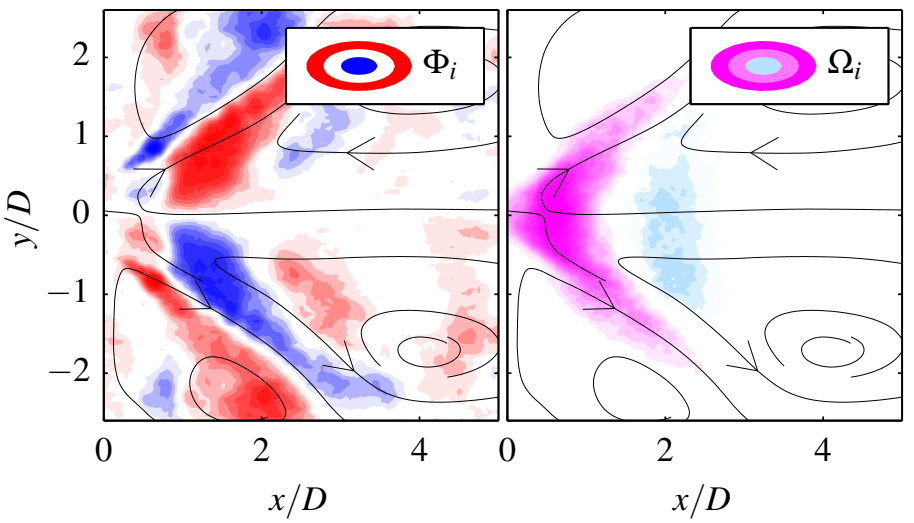

FIGURE 10. SPOD velocity modes $\Phi_{i}$ and corresponding SPOD $\mathrm{OH}^{*}$ modes $\Omega_{i}$ for the shift mode

is reflected by the flame describing function, is governed by different mechanisms, depending on the time scale of the fluctuations. The low frequency fluctuations can be interpreted as slow mean flow dynamics, which are presumably determined by slowly varying inflow conditions. The high frequency fluctuations, instead, correspond to coherent structures driven by the hydrodynamic instability of the underlying mean flow.

Although the application of SPOD is limited to a combustor flow in this presentation, it has high potential to ease the interpretation of data in most fluid dynamic research areas. It allows for the separation of multi-modal dynamics; it provides a very high noise rejection capability, which is very helpful when processing experimental data; it can reconstruct the dynamics of partially recorded phenomena and it provides clean dynamics that can be easily interpreted. These properties make it suited for many applications where the commonly used methods fail to provide insightful results.

\section{ACKNOWLEDGMENT}

Financial supports from the German Research Foundation (DFG) under project grants PA 920/30-1 and from the Research Association for Combustion Engines (FVV) are gratefully acknowledged.

\section{REFERENCES}

[1] Holmes, P., Lumley, J., and Berkooz, G., 1998. Turbulence, Coherent Structures, Dynamical Systems and Symmetry. Cambridge Monographs on Mechanics. Cambridge University Press, Cambridge [England].

[2] Poinsot, T. J., Trouve, A. C., Veynante, D. P., Candel, S. M., and Esposito, E. J., 1987. "Vortex-driven acoustically coupled combustion instabilities". Journal of Fluid Mechanics, 177, 4, pp. 265-292.

[3] Moeck, J. P., Bourgouin, J.-F., Durox, D., Schuller, T., and Candel, S., 2012. "Nonlinear interaction between a precessing vortex core and acoustic oscillations in a turbulent swirling flame". Combustion and Flame, 159(8), pp. 26502668.

[4] Rowley, C. W., Mezic, I., Bagheri, S., Schlatter, P., and Henningson, D. S., 2009. "Spectral analysis of nonlinear flows". Journal of Fluid Mechanics, 641, pp. 115-127.

[5] Schmid, P. J., 2010. "Dynamic mode decomposition of numerical and experimental data". Journal of Fluid Mechanics, 656, pp. 5-28.

[6] Sieber, M., Paschereit, C. O., and Oberleithner, K., 2015. "Spectral proper orthogonal decomposition". Journal of Fluid Mechanics (accepted), preprint arXiv:1508.04642.

[7] Paschereit, C. O., Schuermans, B., Polifke, W., and Mattson, O., 2002. "Measurement of transfer matrices and source terms of premixed flames". Journal of Engineering for Gas Turbines and Power, 124(2), 3, pp. 239-247.

[8] Willert, C., and Gharib, M., 1991. "Digital particle image 
velocimetry". Experiments in Fluids, 10(4), pp. 181-193.

[9] Soria, J., 1996. "An investigation of the near wake of a circular cylinder using a video-based digital cross-correlation particle image velocimetry technique". Experimental Thermal and Fluid Science, 12(2), pp. 221 - 233.

[10] Huang, H. T., Fiedler, H. E., and Wang, J. J., 1993. "Limitation and improvement of PIV: Part II: Particle image distortion, a novel technique". Experiments in Fluids, 15(4-5), pp. 263-273.

[11] Oberleithner, K., Sieber, M., Nayeri, C. N., Paschereit, C. O., Petz, C., Hege, H.-C., Noack, B. R., and Wygnanski, I., 2011. "Three-dimensional coherent structures in a swirling jet undergoing vortex breakdown: stability analysis and empirical mode construction". Journal of Fluid Mechanics, 679, pp. 383-414.

[12] Rukes, L., Sieber, M., Paschereit, C. O., and Oberleithner, K., 2015. "Effect of initial vortex core size on the coherent structures in the swirling jet near field". Experiments in Fluids, 56(10).

[13] Rowley, C. W., Colonius, T., and Murray, R. M., 2004. "Model reduction for compressible flows using POD and galerkin projection". Physica D: Nonlinear Phenomena, 189(12), pp. 115 - 129.

[14] Boree, J., 2003. "Extended proper orthogonal decomposition: a tool to analyse correlated events in turbulent flows". Experiments in Fluids, 35(2), pp. 188-192.

[15] Terhaar, S., Reichel, T. G., Schrödinger, C., Rukes, L., Paschereit, C. O., and Oberleithner, K., 2014. "Vortex Breakdown Types and Global Modes in Swirling Combustor Flows with Axial Injection". Journal of Propulsion and Power, 31(1), pp. 219-229.

[16] Reichel, T. G., Terhaar, S., and Paschereit, O., 2015. "Increasing Flashback Resistance in Lean Premixed SwirlStabilized Hydrogen Combustion by Axial Air Injection". Journal of Engineering for Gas Turbines and Power, 137(7), p. 071503.

[17] Noack, B. R., Afanasiev, K., Morzynski, M., Tadmor, G., and Thiele, F., 2003. "A hierarchy of low-dimensional models for the transient and post-transient cylinder wake". Journal of Fluid Mechanics, 497, 12, pp. 335-363.

[18] Oberleithner, K., Schimek, S., and Paschereit, C. O., 2015. "Shear flow instabilities in swirl-stabilized combustors and their impact on the amplitude dependent flame response: A linear stability analysis". Combustion and Flame, 162(1), pp. $86-99$. 